porter BSEP (18). Thus, the resistance of $\beta$ Klotho-KO mice to gallstones is likely to be a consequence of more than just increased bile acid concentrations in the bile.

One possibility is that the small size of the gallbladder in the $\beta$ Klotho-KO mice reflects changes that contribute to the prevention of gallstone formation. An increase in gallbladder contraction and emptying would be expected to reduce the time that cholesterol has to precipitate and form stones in the bile (19). Indeed, gallbladder stasis is associated with gallstone formation in both humans and rodents $(20$, 21). It will be interesting to learn whether $\beta \mathrm{Klotho}$ is present in the gallbladder and how it affects gallbladder function. Moreover, it will be interesting to see whether FGFR4-KO mice, which also have small gallbladders, are resistant to gallstones.

In summary, the work of Ito and colleagues (2) adds $\beta$ Klotho to the complex tapestry of signaling networks that regulate bile acid homeostasis. Precisely how $\beta$ Klotho integrates with these other regulatory pathways remains to be determined.

Address correspondence to: Steven A. Kliewer, University of Texas Southwestern Medical Center, 5323 Harry Hines Boulevard, Room ND9.502, Dallas, Texas 753909041, USA. Phone: (214) 645-6304; Fax:
(214)645-6305; E-mail: steven.kliewer@ utsouthwestern.edu.

1. Russell, D.W. 2003. The enzymes, regulation, and genetics of bile acid synthesis. Annu. Rev. Biochem. 72:137-174.

2. Ito, S., et al. 2005. Impaired negative feedback suppression of bile acid synthesis in mice lacking BKlotho. J. Clin. Invest. 115:2202-2208. doi:10.1172/ JCI23076.

3. Chiang, J.Y. 2004. Regulation of bile acid synthesis: pathways, nuclear receptors, and mechanisms [review]. J. Hepatol. 40:539-551.

4. Sinal, C.J., et al. 2000. Targeted disruption of the nuclear receptor FXR/BAR impairs bile acid and lipid homeostasis. Cell. 102:731-744.

5. Kerr, T.A., et al. 2002. Loss of nuclear receptor SHP impairs but does not eliminate negative feedback regulation of bile acid synthesis. Dev. Cell. 2:713-720.

6. Wang, L., et al. 2002. Redundant pathways for negative feedback regulation of bile acid production. Dev. Cell. 2:721-731.

7. Kok, T., et al. 2003. Enterohepatic circulation of bile salts in farnesoid $X$ receptor-deficient mice: efficient intestinal bile salt absorption in the absence of ileal bile acid-binding protein. J. Biol. Chem. 278:41930-41937.

8. Holt, J.A., et al. 2003. Definition of a novel growth factor-dependent signal cascade for the suppression of bile acid biosynthesis. Genes Dev. 17:1581-1591.

9. Yu, C., et al. 2000. Elevated cholesterol metabolism and bile acid synthesis in mice lacking membrane tyrosine kinase receptor FGFR4. J. Biol. Chem. 275:15482-15489.

10. Davis, R.A., Miyake, J.H., Hui, T.Y., and Spann, N.J. 2002. Regulation of cholesterol-7alpha-hydroxylase: BAREly missing a SHP [review]. J. Lipid Res. 43:533-543.

11. Gupta, S., Stravitz, R.T., Dent, P., and Hylemon, P.B. 2001. Down-regulation of cholesterol 7alphahydroxylase (CYP7A1) gene expression by bile acids in primary rat hepatocytes is mediated by the c-Jun $\mathrm{N}$-terminal kinase pathway. J. Biol. Chem. 276:15816-15822.

12. Kuro-o, M., et al. 1997. Mutation of the mouse klotho gene leads to a syndrome resembling ageing. Nature. 390:45-51.

13. Ito, S., et al. 2000. Molecular cloning and expression analyses of mouse betaklotho, which encodes a novel Klotho family protein. Mech. Dev. 98:115-119.

14. Admirand, W.H., and Small, D.M. 1968. The physicochemical basis of cholesterol gallstone formation in man. J. Clin. Invest. 47:1043-1052.

15. Tepperman, J., Caldwell, F.T., and Tepperman, H.M. 1964. Induction of gallstones in mice by feeding a cholesterol-cholic acid containing diet. Am.J. Physiol. 206:628-634.

16. Gerloff, T., et al. 1998. The sister of P-glycoprotein represents the canalicular bile salt export pump of mammalian liver. J. Biol. Chem. 273:10046-10050.

17. Smit, J.J., et al. 1993. Homozygous disruption of the murine mdr2 P-glycoprotein gene leads to a complete absence of phospholipid from bile and to liver disease. Cell. 75:451-462.

18. Figge, A., et al. 2004. Hepatic overexpression of murine Abcb11 increases hepatobiliary lipid secretion and reduces hepatic steatosis. J. Biol. Chem. 279:2790-2799.

19. Shaffer, E.A. 1992. Abnormalities in gallbladder function in cholesterol gallstone disease: bile and blood, mucosa and muscle - the list lengthens. Gastroenterology. 102:1808-1812.

20. van Erpecum, K.J., et al. 1992. Fasting gallbladder volume, postprandial emptying and cholecystokinin release in gallstone patients and normal subjects. J. Hepatol. 14:194-202.

21. Wang, D.Q., Schmitz, F., Kopin, A.S., and Carey, M.C. 2004. Targeted disruption of the murine cholecystokinin-1 receptor promotes intestinal cholesterol absorption and susceptibility to cholesterol cholelithiasis. J. Clin. Invest. 114:521-528. doi:10.1172/JCI200416801.

\title{
A molecule's right to choose: how diabetogenic class II MHC products bind peptides
}

\author{
Hidde L. Ploegh \\ Department of Pathology, Harvard Medical School, Boston, Massachusetts, USA.
}

\begin{abstract}
The distinction between peptides that bind to class II MHC products under laboratory conditions and those that do so physiologically is important for the prediction of antigens recognized by autoreactive $T$ cells. In this issue of the JCI, Suri et al., using antigen-presenting cells, compared the peptides that bound to human HLA-DQ8 and those that bound to mouse I-A $\mathrm{A}^{\mathrm{7}}$, both class II MHC products that predispose their carriers to type 1 diabetes (see the related article beginning on page 2268). The rules of engagement for the peptide ligands of the DQ8 and $\mathrm{I}-\mathrm{A}^{\mathrm{g} 7}$ molecules involve similarities in their anchor residues, which mediate stable interaction with class II MHC products. The peptides identified derive from overlapping sets of self proteins.
\end{abstract}

Nonstandard abbreviations used: P9, position 9.

Conflict of interest: The author has declared that no conflict of interest exists.

Citation for this article: J. Clin. Invest. 115:2077-2079 (2005). doi:10.1172/JCI26018.
The single most important genetic factor that predisposes to autoimmune disease is the MHC. In the case of type 1 diabetes, individuals who carry the MHC class II HLA-DQ8 allele are more likely to contract the disease than those who do not, and other alleles at the class II loci (HLA-DR3, HLA-DR4) can predispose to this disease as well. The predominant contribution of the MHC to disease susceptibility is mirrored in the available animal models of autoimmune disease, including diabetes.

In the absence of experiments conducted on humans, the appropriateness of various animal models as stand-ins for human disease continues to be called into question. In mice and humans, the MHC products are very similar in their 3-dimensional structure, although they are no more than $70 \%$ identical in amino acid sequence. Different residues constitute the peptide-bind- 


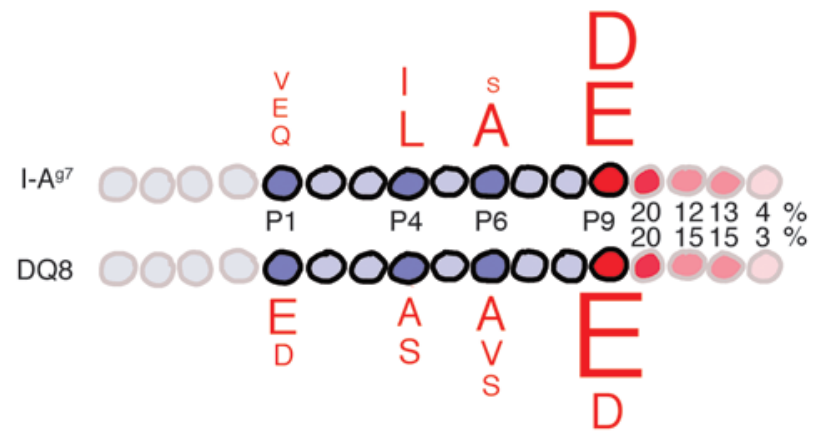

Figure 1

Peptide ligands for the class II MHC products I-A ${ }^{97}$ (mouse) and HLA-DQ8 (human). The central core of the peptide ligand is indicated in bold colors; anchor residues are shown in dark blue (at P1, P4, P6) and red (at P9). The amino acid residues preferred at the key anchor positions are indicated in single letter code, with the size of the letter proportional to the frequency of its occurrence. At P9, acidic residues (glutamic acid [E] and aspartic acid [D]) are universally preferred and account for greater than $90 \%$ of the amino acid residues found at that position. C-terminal of $\mathrm{P9}$, acidic residues (aspartic acid, glutamic acid) are preferred as well. Their frequencies are shown as a percentage of the total number of peptides analyzed that carry an acidic residue at the indicated position. A, alanine; I, isoleucine; L, leucine; $Q$, glutamine; S, serine; V, valine.

ing pocket in the different molecules, and so congruence in the fine specificities of peptide binding would be an unexpected result. Indeed, the peptide sequence motifs that characterize interactions of class I MHC molecules and their peptide ligands reveal little overlap between humans and mice, a feature compounded by the differences in substrate specificity of the human and murine peptide transporters that serve the class I molecules.

\section{Peptides that bind to class II MHC products implicated in type 1 diabetes}

Class II MHC allelic products that carry an aspartic acid residue at position 57 of the $\beta$ chain are protective against type 1 diabetes, whereas the human HLA-DQ8 (hereafter referred to as DQ8) and the NOD mouse class II MHC molecule I-Ag7 lack this particular residue and so predispose to disease. Such predisposition must ultimately be traced to the peptides bound and presented to T cells. In this issue of the JCI, Suri and coworkers now show that the DQ8 and I-Ag7 molecules, notwithstanding their sequence divergence, both engage in very similar modes of peptide binding (1). This binding is specific and concerns many peptides that bind to both the human and the murine class II MHC product. This statement can be made with some confidence, because the class II MHC molecules compared, DQ8 and $\mathrm{I}-\mathrm{A}^{\mathrm{g}}$, were produced in murine cells of identical genetic background. Still, it is not clear to what extent the presence of the murine
$\mathrm{H}-2 \mathrm{M}$ product, involved in dislodging invariant chain remnants and in editing of the class II MHC-bound peptide repertoire, affects the sets of peptides installed in the heterologous DQ8 molecule and might make a contribution to this homogenization.

A major point of debate remains the distinction between the peptides that can bind to MHC products and those that do so under natural circumstances, which may then be recognized in the course of a normal immune response. Spoiled by the availability of predictive algorithms of ever increasing accuracy and the ease of incorporation of peptide ligands into MHC tetramers, those researchers who study class I MHC products have had a good run of it. They can readily verify the presence and measure the frequency of peptide-specific CD8 T cells. Unfortunately, class II MHC molecules are not so readily reconstituted from recombinant subunits, and the numbers of class II tetramers available for analysis of $\mathrm{CD}^{+} \mathrm{T}$ cells pales in comparison to the catalog of class I MHC tetramers. This limits estimates of the frequencies with which peptide-specific CD4 ${ }^{+} \mathrm{T}$ cells occur, and clearly we need accurate information on naturally processed peptides complexed with class II MHC molecules.

The work of Suri et al. (1) now provides a comprehensive data set for peptides bound to the diabetogenic class II MHC products DQ8 and I-Ag7. The authors show, first, that alternative strategies used earlier to identify potential binders don't necessarily yield the naturally processed peptides. Any predictive or experimental scheme that fails to take into account what antigen-presenting cells are capable of producing will confound the picture. In passing, it should be noted that even naturally processed peptides that bind to MHC products may well be invisible from a mature T cell's perspective: the class I ligand FAPGNYPAL is presented by $\mathrm{H}-2 \mathrm{~K}^{\mathrm{b}}$ in Sendai virus-infected cells, but, notwithstanding the ability of $\mathrm{H}-2 \mathrm{D}^{\mathrm{b}}$ to bind this peptide strongly, no $\mathrm{H}-2 \mathrm{D}^{\mathrm{b}}$-restricted, FAPGNYPAL-restricted $\mathrm{CD}^{+} \mathrm{T}$ cells seem to be activated in the course of a Sendai virus infection (2). There is no reason to assume the situation would be different for class IIrestricted responses.

Suri and coworkers not only compiled a catalog of naturally processed peptides but also provide an in-depth analysis of their mode of binding (1). The good news is that the DQ8 and I-A ${ }^{97}$ molecules march to the same drumbeat where peptide binding is concerned: similar source antigens, similar anchor residues, and hence similar peptides are presented. This should be a source of considerable relief to those who study the NOD mouse as a model for human type 1 diabetes. There are discrepancies between the pools of peptides identified as being able to bind to class II MHC molecules by phage display and peptide library-based methods and those retrieved as naturally processed fragments. Consequently, the architecture of the peptide-binding pocket alone cannot account for the remarkable convergence of peptides bound by DQ8 and $\mathrm{I}-\mathrm{A}^{\mathrm{g}}$. The most striking feature of this interaction is the near omnipresence of an acidic residue at position 9 (P9) (Figure 1) for both DQ8 and I-Ag7 ligands. In addition, there is what appears to be a highly significant clustering of such acidic residues C-terminal to P9. These acidic residues contribute to interactions outside of the peptide-binding groove proper.

\section{Other factors that contribute to the generation of class II MHC-peptide complexes}

A robust method for analyzing naturally processed peptides will be very useful in dissecting the complex response against pancreatic self antigens. In humans, presentation of diabetogenic epitopes is of course not limited to the DQ8 molecule, as evidenced by the recently identified DR4restricted insulin A 1-15 epitope (3).

What these approaches cannot take into consideration is the tortuous pathway by which class II MHC molecules reach the 
destination where newly generated peptide pools are made available for binding to them. Absent from the discussion is the obvious consideration of protease specificity: if lysosomal proteases that generate the class II MHC ligands were to display preferred cleavage sites, then the pools of peptides made available to class II molecules would be biased. However, this has never been a popular notion in view of the redundancy, in terms of substrate specificity, of lysosomal proteases. There are examples to the contrary, however: asparagine-specific endoprotease is indeed remarkably selective in the peptide bonds it cleaves, and the enzyme has been implicated in the generation, in a highly selective fashion, of class II MHC-restricted epitopes (4). Nonetheless, there are very few examples of antigens for which the processing pathway has been characterized comprehensively in terms of intermediates and the proteases that generate them. It may well be that the convergence in peptide binding, observed for DQ8 and I-Ag7, produced by the identical types of antigen-presenting cells, owes at least a component of this repertoire to shared enzymatic machinery upstream. As Suri et al. state (1), expression levels of the source antigen and the possibilities of encounter with the entire processing and peptide loading apparatus are certainly going to affect the final outcome, in terms of the MHC-peptide complexes produced. The best map of a landscape is the landscape itself: seen from this perspective, the comprehensive data set presented here by Suri and coworkers will allow others to navigate the territory of autoimmunity with an accurate map in hand.
Address correspondence to: Hidde L. Ploegh, Department of Pathology, Harvard Medical School, 77 Avenue Louis Pasteur, Boston, Massachusetts 02115, USA. Phone: (617) 432-4776; Fax: (617) 432-4775; E-mail: ploegh@hms.harvard.edu.

1. Suri, A., Walters, J.J., Gross, M.L., and Unanue, E.R. 2005. Natural peptides selected by diabetogenic DQ8 and murine I-Ag7 molecules show common sequence specificity. J. Clin. Invest. 115:2268-2276. doi:10.1172/JCI25350.

2. Kast, W.M., et al. 1991. Protection against lethal Sendai virus infection by in vivo priming of virus-specific cytotoxic $T$ lymphocytes with a free synthetic peptide. Proc. Natl. Acad. Sci. U. S. A. 188:2283-2287.

3. Kent, S.C., et al. 2005. Expanded T cells from pancreatic lymph nodes of type 1 diabetic subjects recognize an insulin epitope. Nature. 435:224-228.

4. Antoniou, A.N., Blackwood, S.L., Mazzeo, D., and Watts, C. 2000. Control of antigen presentation by a single protease cleavage site. Immunity. 12:391-398.

\title{
Of mice and men: the iron age
}

\author{
Sophie Vaulont, Dan-Qing Lou, Lydie Viatte, and Axel Kahn
}

Institut Cochin, INSERM 567, CNRS 8104, Université Paris 5, Paris, France.

\begin{abstract}
Recently, mutations causing juvenile hemochromatosis have been identified in a novel gene, hemojuvelin (HJV), located on chromosome 1. Mouse models of this disease have now been developed by 2 groups, Huang et al. and Niederkofler et al., through targeted disruption of the $\mathrm{Hj} v$ gene (see the related articles beginning on pages 2180 and 2187). These mutant mice will allow further investigation into the role of $\mathrm{HJV}$ in the regulation of iron homeostasis, a role that to date remains elusive.
\end{abstract}

The following scenario is classic with respect to genetic disorders: the disease is clinically recognized, the related gene is identified, and a couple of years later, a mouse model of the genetic disease is established. The genes involved in iron metabolism have not been an exception to this rule (in particular those related to hereditary hemochromatosis, a prevalent heterogeneous iron-overload disorder), and the development of mouse models have greatly facilitated our understanding of the pathogenesis of iron overload. The hemojuvelin gene (HJV, also known as HFE2 and RGMc), which is mutated in a

Nonstandard abbreviations used: HAMP, hepcidin antimicrobial peptide; HJV, hemojuvelin; TFR2, transferrin receptor 2 .

Conflict of interest: The authors have declared that no conflict of interest exists.

Citation for this article: J. Clin. Invest. 115:2079-2082 (2005). doi:10.1172/JCI25642. form of juvenile hemochromatosis linked to chromosome 1, was identified in 2003 (1). Researchers in the field have therefore been waiting for the development of a relevant mouse model of this disease. This feat has now been accomplished by Huang et al. and Niederkofler et al., who in this issue of the JCI describe the phenotype of their mutant mice $(2,3)$. Their findings add further support to the emerging attractive hypothesis that hepcidin, a recently described iron-regulatory peptide, and ferroportin, its target, are common pathogenic denominators in all forms of iron overload.

In mammals, iron homeostasis is complex and depends on regulated dietary iron absorption by mature enterocytes of the duodenum and iron recycling by macrophages, which supply most of the serum iron through recovery of the metal from senescent erythrocytes (4). These 2 fundamental processes are deregulated in hereditary hemochromatosis; over time, this deregulation leads to iron deposition in parenchymal cells and transferrin saturation (5). As the disease progresses, patients develop iron-induced tissue damage, which results in serious illnesses including cirrhosis, hepatomas, diabetes, cardiomyopathy, arthritis, and endocrinopathies. Unless recognized and treated, this disease is fatal.

While hereditary hemochromatosis has been clinically recognized since 1889 , it is only over the 10 past years that the genetic basis for the disease has been elucidated. Hereditary hemochromatosis is now recognized to be genetically heterogeneous and its nomenclature is under debate. Depending on distinct clinical and genetic entities, hereditary hemochromatosis can be divided into 3 classes: classical hemochromatosis, juvenile hemochromatosis, and ferroportin disease.

Classical hemochromatosis is associated with the historical and most prevalent form of hereditary hemochromatosis and is almost always caused by mutations in $H F E$, a gene which encodes a protein of the major histocompatibility complex class I (6). Rarely, another gene can be involved, namely transferrin receptor 2 (TFR2) (7), which encodes a homolog of TFR1 that mediates 